\title{
Gerlinde Huber-Rebenich (dir.), Lehren und Lernen im Zeitalter der Reformation
}

\section{Aude-Marie Certin}

\section{OpenEdition}

\section{Journals}

Édition électronique

URL : http://journals.openedition.org/ifha/8737

DOI : $10.4000 /$ ifha. 8737

ISSN : 2198-8943

\section{Éditeur}

IFRA - Institut franco-allemand (sciences historiques et sociales)

\section{Référence électronique}

Aude-Marie Certin, « Gerlinde Huber-Rebenich (dir.), Lehren und Lernen im Zeitalter der Reformation », Revue de l'IFHA [En ligne], Date de recension, mis en ligne le 02 novembre 2017, consulté le 24 septembre 2020. URL : http://journals.openedition.org/ifha/8737 ; DOI : https://doi.org/10.4000/ifha. 8737

Ce document a été généré automatiquement le 24 septembre 2020.

(C)IFHA 


\title{
Gerlinde Huber-Rebenich (dir.), Lehren und Lernen im Zeitalter der Reformation
}

\author{
Aude-Marie Certin
}

\section{RÉFÉRENCE}

Gerlinde Huber-Rebenich (dir.), Lehren und Lernen im Zeitalter der Reformation, Tübingen: Mohr Siebeck (Spätmittelalter, Humanismus, Reformation 68), 2012, $89 €$

Ce livre collectif porte sur les thèmes de l'enseignement et de l'apprentissage au temps de la Réforme. Il est le fruit d'un colloque qui s'est tenu en 2009 à la bibliothèque de recherches Gotha et venait conclure une exposition consacrée à Mutianus Rufus (1471-1526), figure non conventionnelle de l'humanisme à Erfurt. Lors de cette rencontre, les participants, dont les contributions sont rassemblées dans ce présent volume, se sont interrogés sur les différentes formes de transmission des savoirs à l'époque de Mutianus Rufus et jusqu'après la Réforme, au moment de la fixation des formes d'enseignement. Les articles de ce volume envisagent non seulement les contenus transmis mais aussi, de façon plus générale, les méthodes de transmission et la visée des apprentissages. Y sont étudiées les matières enseignées, les institutions impliquées (du cloître à l'université) ainsi que les différentes formes de discours éducatifs. Ralf Koerrenz y étudie certains écrits pédagogiques de Luther en montrant que ce dernier donne à l'école en tant qu'institution une importance cruciale dans l'éducation publique. Ainsi constitue-t-elle un défi essentiel pour les protestants de son époque. Harald Müller s'intéresse pour sa part à la correspondance de l'humaniste Nikolaus Ellenbog (1481-1543) sous l'angle des recommandations d'apprentissage et de lecture faites à des religieuses. Michael Rupp étudie les différents usages du latin chez Paulus Niavis en soulignant les différents régimes de langue qu'il utilisait en fonction des situations et des publics. Michael Baldzuhn s'interroge sur ce qui était transmis et 
enseigné dans les cours de latin en reprenant les recommandations et les réflexions d'Érasme en ce domaine.

Walther Ludwig étudie la forme et la visée des méthodes d'enseignement déployées par Mélanchton dans l'Initia doctrinae physicae de 1549. Partant de la disputation de Leipzig de 1519 et de celle de Zurich de 1523, Volker Leppin montre comment cette forme de discussion universitaire conventionnelle est devenue un médium essentiel de la réforme de l'Eglise en se diffusant hors du cadre académique dans l'espace public. Thomas Töpfer étudie la dimension didactique des Loci communes de Philipp Mélanchton et sa postérité après la mort de son auteur. Daniel Gehrt se penche sur l'importance du Kleinen Corpus Doctrinae de Matthäus Judex dans le contexte de la Réforme et sur sa diffusion jusqu'au XVII ${ }^{e}$ siècle. Franz Körndle s'intéresse aux formes d'enseignement de la musique aux XV $\mathrm{XV}^{\mathrm{e}}$ et XVI $\mathrm{XI}^{\mathrm{e}}$ siècles et au vocabulaire utilisé, en allemand et en latin, dans le monde académique et en dehors. Dans une autre contribution portant sur l'époque de la Contre-Réforme, il s'intéresse également à des morceaux de musique et à des chants de l'ordre des Jésuites à partir de sources méconnues. Christel Meier-Staubach présente quant à elle un projet de recherche sur les formes d'enseignement du théâtre au début de l'époque moderne, à la fois dans le milieu protestant, dans différents ordres, mais aussi dans les cours et en ville. Sans prétendre traiter le thème de l'éducation au temps de la Réforme de façon exhaustive, cet ouvrage collectif a ainsi le grand intérêt d'apporter des éclairages divers et inédits sur cette thématique d'ensemble.

INDEX

Thèmes : Histoire sociale, Histoire de la culture, Histoire des mentalités, Histoire des idées Index chronologique : Période moderne 